\title{
New Opportunities for Quantitative Tracking of Polycrystal Responses in Three Dimensions
}

Jay C. Schuren ${ }^{1, \wedge}$, Paul A. Shade ${ }^{1,{ }^{*}}$, Joel V. Bernier ${ }^{2}$, Shiu Fai $\mathrm{Li}^{2}$, Basil Blank ${ }^{3}$, Jonathan Lind ${ }^{2,6}$, Peter Kenesei ${ }^{4}$, Ulrich Lienert ${ }^{5}$, Robert M. Suter ${ }^{6}$, Todd J. Turner ${ }^{1}$, Dennis M. Dimiduk ${ }^{1}$, Jonathan Almer ${ }^{4}$

${ }^{1}$ Materials and Manufacturing Directorate, Air Force Research Laboratory, Wright-Patterson AFB, OH 45433, USA

${ }^{2}$ Engineering Directorate, Lawrence Livermore National Laboratory, Livermore, CA 94550, USA

${ }^{3}$ PulseRay, Beaver Dams, NY, 14812, USA

${ }^{4}$ Advanced Photon Source, Argonne National Laboratory, Argonne, IL, 60439, USA

${ }^{5}$ DESY-Petra III, Hamburg, Germany

${ }^{6}$ Carnegie Mellon University, Pittsburgh, PA, 15213, USA

${ }^{*}$ To whom correspondence should be addressed, E-mail: paul.shade.1@us.af.mil

^Present address: Nutonian Inc., Somerville, MA, 02144, USA

\begin{abstract}
An important advance in understanding the mechanics of solids over the last 50 years has been development of a suite of models that describe the performance of engineering materials while accounting for internal fluctuations and anisotropies (ex., anisotropic response of grains) over a hierarchy of length scales. Only limited engineering adoption of these tools has occurred, however, because of the lack of measured material responses at the length scales where the models are cast. Here, we demonstrate an integrated experimental capability utilizing high energy X-rays that provides an in situ, micrometer-scale probe for tracking evolving microstructure and intergranular stresses during quasi-static mechanical testing. We present first-of-a-kind results that show an unexpected evolution of the intergranular stresses in a titanium alloy undergoing creep deformation. We also discuss the expectation of new discoveries regarding the underlying mechanisms of strength and damage resistance afforded by this rapidly developing $\mathrm{X}$-ray microscopy technique.
\end{abstract}

\section{Introduction}

Many critical engineering materials, such as metals and ceramics, are polycrystalline. As such, they are inherently heterogeneous over a hierarchy of length scales ranging from the engineered component, to the distribution of different crystalline grains, phases, voids and/or secondary particles, and down to the distribution of lattice defects at the atomic scale. This structural heterogeneity manifests as anisotropy in functional material properties such as stiffness, strength, conductivity, damage resistance, etc. However, engineered products are almost universally designed using idealized homogeneous representations of the material at the macroscopic scale. While this approximation scheme permits unambiguous linkages between boundary condition constraints and material response for a range of structures such as bridges, aircraft, and electronic devices, it is often quite limited in terms of predictive capability.

More recently, much interest has been paid to materials science at the so called "mesoscale" [1,2]. While not an explicit length scale, the mesoscale can be thought of here as a 
length scale over which homogenization breaks down, and where materials properties are the result of an ensemble of constituents. In the present context, this length scale encompasses an ensemble of individual crystallites or "grains", possibly including a distribution of voids and secondary phase particles. Each grain or constituent expresses directionally dependent properties (e.g. strength, stiffness, electrical conductivity, etc.) stemming from the atomic compositions and structure along with crystal defects. The metrics of these ensembles, referred to as the microstructure of the material, result in the internal forces of a loaded body being distributed quite heterogeneously. In turn, these heterogeneities drive the emergent behaviors of creep, crack initiation and fracture. With few exceptions, mesoscale information has not been incorporated into the design process, not because mesoscale modeling tools don't exist, but rather because of a lack of appropriate experimental data and methods with which to validate such models, leaving an unacceptable level of risk for a design engineer.

One approach toward capturing the necessary experimental data is integration of in situ mechanical testing with advanced characterization methods to provide full 3D microstructural characterization of the test volume, in which ensembles of individually resolved grains are tracked throughout an experiment [3]. Measuring a sufficiently large number of grains is important, not only to characterize the heterogeneities in internal stresses as they develop, but also to facilitate detection of rare events such as void/crack nucleation. This information could be used to validate predictive models that explicitly represent 3D microstructure [4]. In this work, we describe a novel capability to nondestructively characterize the evolving microstructure and micromechanical state of deforming polycrystalline ensembles through concurrent integration of three high energy synchrotron X-ray techniques. We present first-ofa-kind results for a titanium alloy specimen undergoing time dependent creep deformation that reveal a complex redistribution of internal stresses during the creep process.

\section{Methods}

\subsection{High energy diffraction microscopy (HEDM)}

The enabling technology for performing the measurements presented herein is highenergy synchrotron radiation. High-energy synchrotron sources provide a unique blend of high brilliance, high-energy $(>50 \mathrm{keV})$ radiation that enables nondestructive evaluation of microstructure and micromechanical state in bulk (penetration depths on the order of millimeters) engineering materials. For reference, the brilliance of these light sources can be 610 orders of magnitude greater than that of a laboratory X-ray source. Over the past 15 years, several experimental techniques capable of independently mapping structure and grain-bygrain mechanical response have been developed. In order to study "bulk" phenomena, as well as the relevant statistics to capture critical events (void/crack nucleation), it is necessary to measure on the order of 1000 grains. The most prevalent techniques capable of spatially resolved measurements of microstructure and micromechanical state for aggregates that large are tomography and a class of diffraction-based measurements that are built upon the rotating crystal method $[5,6]$. These techniques consist of interrogating a sample with monochromatic $X$-rays while the sample is continuously rotated and images of diffracted beams are collected on area detectors in transmission geometry over discrete angular intervals [7-10], and are alternately referred to as three-dimensional X-ray diffraction microscopy (3DXRD) or highenergy X-ray diffraction microscopy (HEDM). These techniques differ from polychromatic 
methods such as differential aperture X-ray microscopy (DAXM) [11], which consists of pointby-point measurements and uses micro-focused beams of lower energy polychromatic X-rays (8-35 keV) to probe three dimensional structure relatively near sample surfaces. The polychromatic methods offer excellent intragranular resolution, but are less suited for characterizing large ensembles of interior grains.

The three X-ray techniques we have integrated are referred to as far-field HEDM (ffHEDM) [12-15], near-field HEDM (nf-HEDM) [8,16,17], and absorption micro-computed tomography $(\mu-\mathrm{CT})[18]$. These techniques result in correlated data that (1) quantify an average elastic strain tensor (stress tensor with known elastic stiffness matrix) for each grain, (2) map the structure and local crystallographic orientation within and between grains, and (3) permit observation of the structure of voids, cracks, and second phase inclusions, respectively. In each case, raw data consists of images of diffracted or transmitted X-ray beams collected on area detectors placed at different working distances while the specimen is rotated and irradiated with a monochromatic X-ray beam. The experiment was conducted the high energy beamline 1-ID-E at the Advanced Photon Source (APS), Argonne National Laboratory using an X-ray energy of $65.4 \mathrm{keV}$. While measurement hardware, procedures, and analysis software have been developed independently for each technique, the ability to collect unified correlated datasets yields a more complete view of the evolving material that is greater than the sum of the independent results.

\subsubsection{Far field high energy diffraction microscopy (ff-HEDM):}

The ff-HEDM technique provides the average elastic strain tensor (from which average stress tensors can be calculated assuming linear elasticity), the average crystallographic orientation, and the center of mass position for individual grains within a deforming polycrystalline sample [12-15]. The data reduction consists of a back-projection method where individual diffraction spots are first identified by segmentation of the detector image, subsequently associated with one or more Debye-Scherrer rings using a specified space group and angular tolerance, then finally associated with a uniquely oriented crystal lattice through an indexing operation. Once a set of orientations is obtained, the 12 degrees of freedom that describe the orientation (3), position (3), and elastic strain (6) of an individual grain are optimized, in a least-squares sense, using the subset of measured spot centroids associated with it from the indexing [15]. The detector is positioned "far" ( 1 $\mathrm{m})$ from the specimen to improve angular resolution of the diffraction pattern and thus provide high elastic strain sensitivity. Typically the strain resolution of this technique is quoted to be $\pm 1 \times 10^{-4}$. This value is directly coupled to experimental conditions and is often conservative $[19,20]$. It is important to note that these measurements differ from conventional 'aggregate' or so called powder experiments since each diffraction spot originates from and is assigned to an individual grain within the specimen while accounting for precession during the rotation of the specimen.

The selection of the $\mathrm{X}$-ray beam size with respect to the average grain size in the specimen dictates how the ff-HEDM results should be interpreted. If the beam size is large enough such that the grains of interest are fully irradiated, then the center of mass, average orientation, and the average elastic strain tensor represent grain averaged quantities. However, in the current work we used a line focused X-ray beam as wide as the specimen ( $1.4 \mathrm{~mm}$ on the diagonal) but only $\sim 2 \mu \mathrm{m}$ tall (along the tensile axis). The novel application of the line 
focused beam for the ff-HEDM technique provides the average elastic strain and stress tensors for the irradiated portion of each grain, i.e. the grain cross-section averaged (GCSA) elastic strains/stresses, resulting in sub-grain resolution in the direction orthogonal to the plane of the beam.

\subsubsection{Near field high energy diffraction microscopy (nf-HEDM)}

The nf-HEDM experiment nondestructively characterizes the microstructure (crystallographic orientation, size/shape/relative position of each grain) within the diffracting sample $[8,16,17]$. The measurement presented herein employed the same line focused X-ray beam as for ff-HEDM ( $2 \mu \mathrm{m}$ by the width of the sample) to scan a 'layer' of the material. A high resolution detector images the shapes of diffraction spots as the sample rotates. A specimen volume is mapped by translating the sample perpendicular to the beam plane to illuminate successive layers. Diffraction measurements from each layer are measured at multiple sample to detector distances such that diffraction spots are seen to radiate away from the grain of origin, thus encoding the grain position as well as the scattering angles. The salient feature of the experimental geometry is that the detector needs to be "near" the specimen (within 10 $\mathrm{mm}$ ), providing greater sensitivity to grain position rather than diffraction angle. The data reduction consists of iteratively comparing a forward model of potential crystal orientations for each volume element (voxel) within the sample to the measured diffraction patterns $[16,17]$. For nf-HEDM the orientation and spatial resolution are often quoted as $<0.1^{\circ}$ and $2 \mu \mathrm{m}$, respectively. Given the sample to detector distance requirements for nf-HEDM, concurrent application of the technique during conventional mechanical testing has previously not been possible. Application of a conventional loadframe on a rotation stage would result in the support posts striking the nf-HEDM detector during rotation.

\subsubsection{Absorption micro-computed tomography ( $\mu$-CT)}

The $\mu$-CT measurements were collected with a beam hundreds of microns tall which spanned the width of the sample cross-section. This technique relies on imaging variations in the intensity of the direct transmitted beam during rotation of the sample. This information corresponds to fluctuations of electron density in the material and can be used to identify and track the evolution of voids, cracks, and additional phases or inclusions [18]. This technique provides key information for identifying regions of interest and monitoring the evolution during application of external stimuli. The resolution of $\mu$-CT is defined by the experimental conditions, but $<1 \mu \mathrm{m}$ has become commonplace for lower energy X-rays. With the experimental conditions employed here using high energy $X$-rays, the detector resolution of $\sim 1.5 \mu \mathrm{m}$ sets the measurement resolution.

\subsection{Rotation and linear axial motion system (RAMS)}

The concurrent application of $\mu-C T$, ff-HEDM, and nf-HEDM during loading requires the precise $360^{\circ}$ rotation of a sample undergoing tensile and/or compressive loading within the grips of a mechanical testing system (loadframe) without obstructing the $\mathrm{X}$-ray signal or operation of the X-ray detectors. Therefore, we have made a major advance by developing a high precision coaxial two-spool rotation and linear axial motion system (RAMS) [21], as shown 
in Figure 1. The RAMS device was designed to overcome the constraints imposed by the proximity of the nf-HEDM detector and allows the specimen to rotate continuously within the grips of a stationary conventional mechanical testing system while undergoing tensile and/or compressive loading up to $2 \mathrm{kN}$. The RAMS device uses a paired spool design, where each spool consists of two air bushings (center of the spool) to carry radial loads and two air bearings (top/bottom of the spool) to carry axial loads. This allows for precise angular motion from small steps to continuous rotation about the loading axis. Rotation of the specimen during uniaxial loading is achieved through a servomotor, gear reducer, preloaded linear ball spline, and two timing belts.

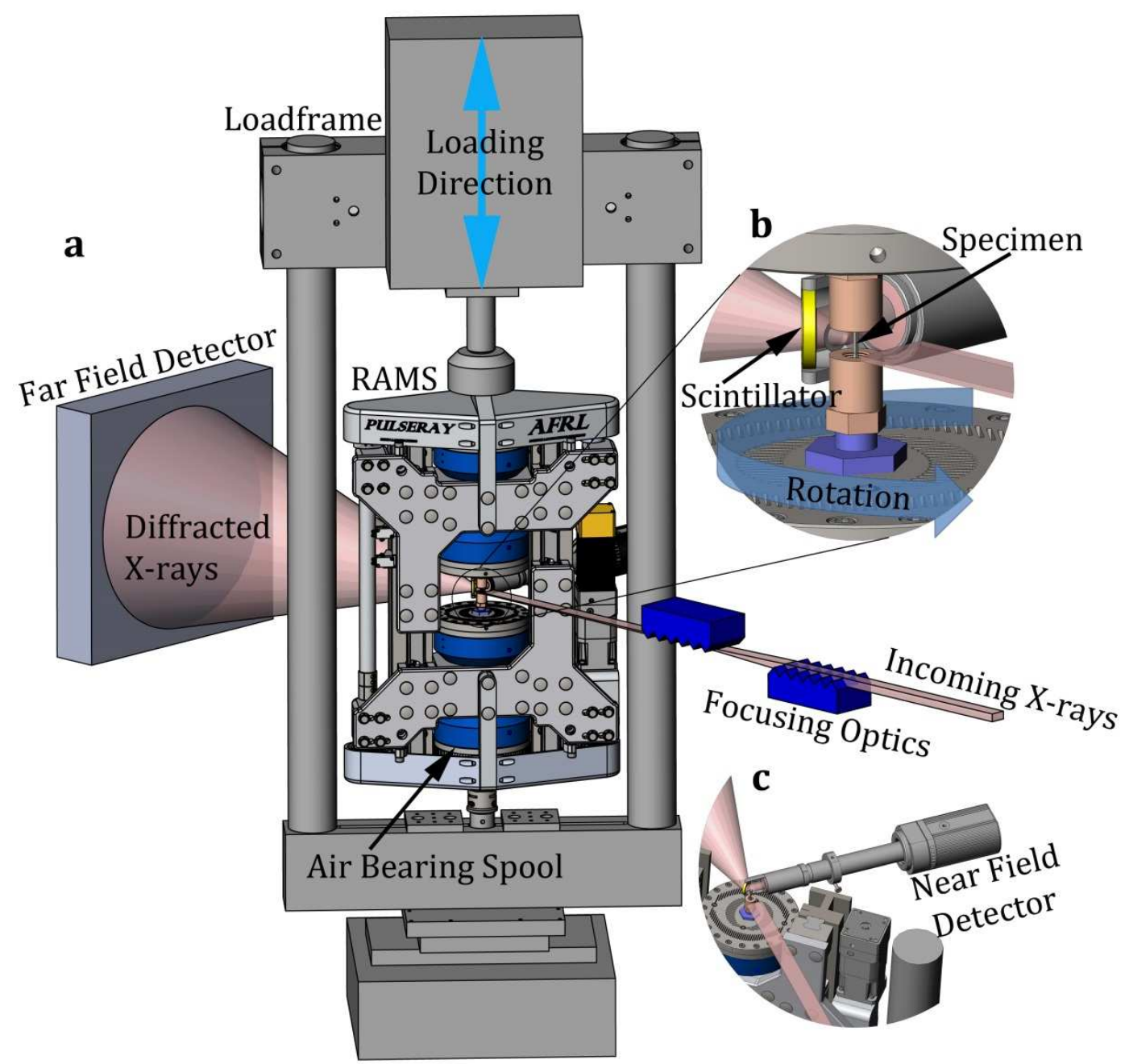

Figure 1. Experimental setup with various aspects shown to scale. (a) A schematic illustration of the rotation and linear axial motion system (RAMS), loadframe, and the far field detector are shown with respect to the X-ray beam. (b) A typical specimen mounted within the grips is shown to indicate the close proximity to the near field detector scintillator. (c) A cut away "topview" of the RAMS device illustrates that, while $360^{\circ}$ rotation of the entire loadframe is not possible due to conflict with the near field detector, the RAMS device achieves this rotation within the loadframe grips. 
The RAMS device was mounted within the grips of a servohydraulic MTS model 858, available at the APS 1-ID beamline. Each subassembly was designed to support the axial and radial loads expected during the mechanical test. Moreover, since we seek to measure the precession of diffracting grains, it is imperative that any eccentricity or non-recoverable wobble in the rotation system be minimized. In conventional systems where a loadframe is positioned on a rotation stage, this artifact is introduced due to the rotation axis and the loading direction not being exactly parallel, which limits the overall resolution of each of the techniques. Using a capacitance measurement system we determined the non-recoverable wobble within the RAMS to be below the resolution of each technique.

\subsection{Material}

In a first application of this new capability, we measured a titanium alloy (Ti-7Al) tensile specimen undergoing room temperature creep deformation. The Ti-7Al material (provided by Dr. Adam Pilchak, AFRL) was initially cast as a $\sim 75 \mathrm{~mm}$ ingot, hot isostatic pressed, extruded into a $\sim 30 \times 30 \mathrm{~mm}^{2}$ square, and finally unidirectionally rolled at $955^{\circ} \mathrm{C}$ to a thickness of $\sim 7.5$ $\mathrm{mm}$. The material was recrystallized at $955^{\circ} \mathrm{C}$ for 24 hours and furnace cooled, producing a single phase $\alpha$ (HCP crystal structure) material with a basal texture and nearly-equiaxed grains with an average size of $\sim 100 \mu \mathrm{m}$. The tensile specimen was prepared with the tensile axis transverse to the rolling and normal directions. The anisotropic elastic moduli employed to convert elastic strains to stresses are shown in Table 1.

\begin{tabular}{lclcl}
\hline$C_{11}$ & $C_{12}$ & $C_{13}$ & $C_{33}$ & $C_{44}$ \\
\hline 162,400 & 92,000 & 69,000 & 180,700 & 46,700 \\
\hline
\end{tabular}

Table 1. The elastic constants (Voigt convention) used for this effort are shown in units of MPa [22].

Creep is the time dependent plastic deformation of a material under constant load. While typically thought of as an elevated temperature behavior, titanium alloys are well known to exhibit significant creep at room temperature [23]. Titanium creep behavior is a complex phenomenon dependent on the local microstructure and grain level interactions, among other factors, and is an area of interest because it has been tied to dwell fatigue sensitivity which is a major design constraint for titanium based structural components [24-26].

\section{Results and discussion}

\subsection{Experiment}

The specimen was held in the RAMS device and subjected to an applied tensile load of $525 \mathrm{MPa}$ (near the elastic limit), then the load was immediately reduced to $473 \mathrm{MPa}$ and held fixed for 24 hours. In addition to the elastic strains accumulated during loading, the specimen accumulated $\sim 0.6 \%$ plastic strain during the creep hold. All three high energy X-ray measurements were carried out on the same 3D region of the sample, while under load, and the output data sets have been put into a common reference frame. While a complete interpretation of the material evolution is beyond the scope of this paper, we demonstrate the 
richness of the combined data sets and show that novel observed phenomena can be extracted from only a single layer of the reconstructed volume.

\subsection{Structural characterization}

The 3D grain structure obtained from in-situ nf-HEDM of a subsection of the specimen is shown in Figure 2a. The angle between the applied loading direction and the local crystallographic 'c-axis', which influences slip and twinning responses, is shown in Figure $2 \mathrm{~b}$. Figure $2 \mathrm{c}$ shows a layer from a $\mu$-CT measurement, which was used to measure and track the cross-sectional area of the specimen in order to convert the applied load to a macroscopic tensile stress value. The $\mu$-CT scan also showed that the sample was fully dense with no voids, cracks, etc. at dimensions above the $\sim 1.5$ micron measurement resolution.

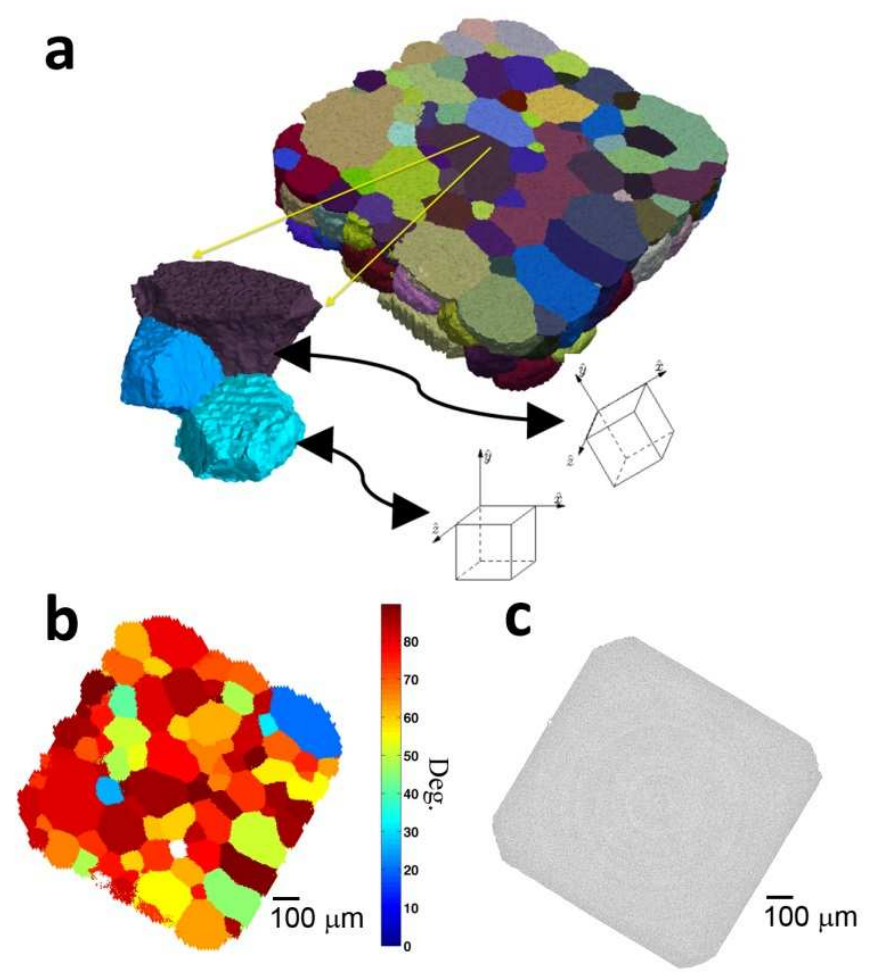

Figure 2. Structure of Ti-7Al tensile specimen. (a) A $1 \times 1 \times 0.192 \mathrm{~mm}^{3}$ volume of the specimen as measured with nf-HEDM is shown with each grain colored by the crystallographic orientation using each degree of freedom in the Rodrigues orientation parameterization (see [27]) to set the RGB color value. Three grains are isolated to convey the $\sim 2 \mu \mathrm{m}$ resolution of the reconstructed boundaries. (b) The co-axiality angle between the crystallographic c-axis and loading direction is shown for each grain in a single cross section. (c) Layer from a $\mu$-CT measurement showing the sample cross-section. This measurement confirmed that the specimen gauge volume was absent of voids or cracks.

\subsection{Merging data from multiple techniques}

Merging of the nf-HEDM and ff-HEDM datasets begins with the assumption that the same region of the specimen is irradiated in both measurements. We used the same line 
focused beam for both but the data were collected sequentially and this may lead to micron scale thermal or other drifts (future work will implement simultaneous collection in each layer). Center of mass positions and average orientations for each grain can be used to align datasets but this was not necessary in the present case. On the other hand, drifts may contribute to the few discrepancies that are found in the sets of grains identified by nf-HEDM and ff-HEDM analysis, as shown in Figure 3. For example, small cross-sections associated with grain edges may come into or out of the beam. Further, due to differing resolutions of the two techniques set by the pixel sizes and the very different dynamic ranges of the detectors, there may be cases where small cross-sections not visible to the nf-HEDM are identified by ff-HEDM.

While nf-HEDM and ff-HEDM data were measured sequentially in time, they were both measured concurrently with respect to the deformation. Throughout the deformation, the merging of the nf-HEDM, ff-HEDM, and $\mu$-CT results is facilitated through the use of a fiducial marker. A $30 \mu \mathrm{m}$ gold cube was bonded to the specimen surface to provide a non-deforming reference feature.

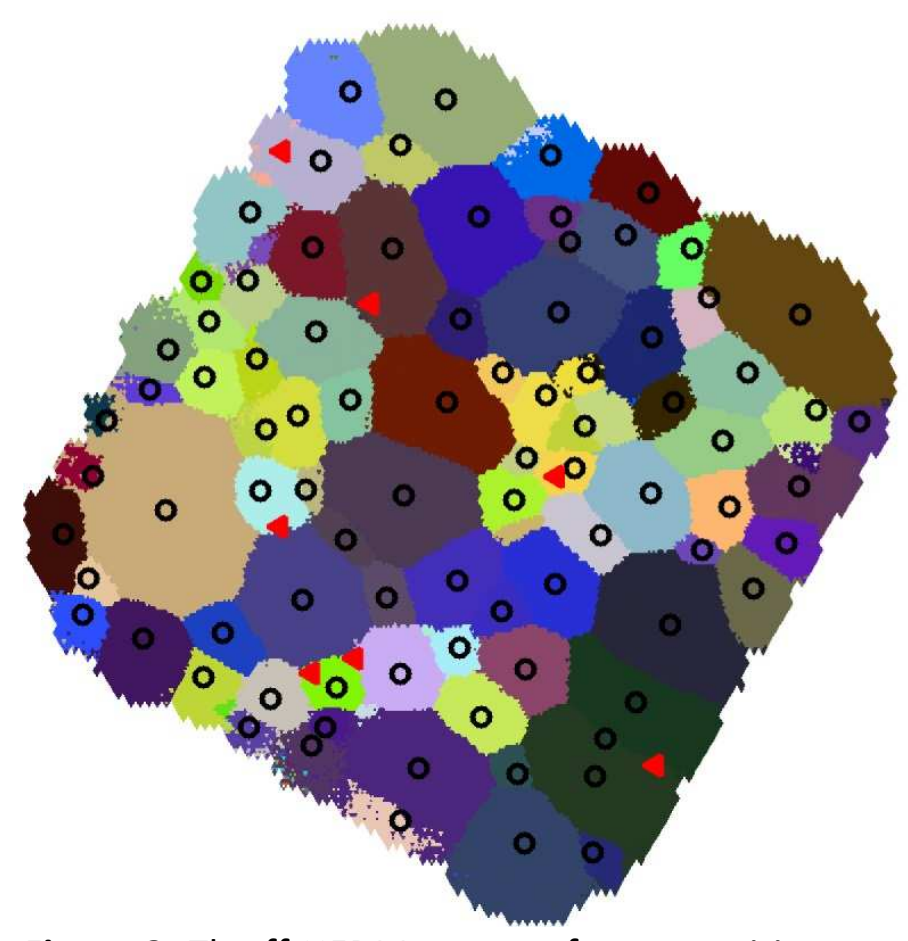

Figure 3. The ff-HEDM center of mass positions are overlaid as symbols on the nf-HEDM grain map. The red triangles are small grains identified by ff-HEDM that were not present in the nfHEDM analysis.

\subsection{Completeness}

The primary figure of merit in both the nf-HEDM and ff-HEDM data reduction is alternately referred to as "confidence" or "completeness" $[15,17]$. It is calculated as the ratio of indexed (measured) to predicted diffraction spots on the detector for each single-lattice scattering unit subject to an appropriate set of tolerances. For the ff-HEDM analysis we present results with a completeness greater than $40 \%$. For the ff-HEDM experimental conditions presented here there were 216 expected diffraction spots and the minimum number measured 
for a grain above the $40 \%$ completeness threshold was 86 . The white regions shown in Figure $2 \mathrm{~b}$, Figure 6 and Figure 8 are not voids, but rather regions where the completeness of the ffHEDM data reduction is below this threshold. This is not a limitation of the data, but rather highlights the further need for developing data reduction tools built upon a forward model as opposed to the back-projection approach previously described.

Completeness maps for the nf-HEDM and ff-HEDM results are shown in Figure 4 and Figure 5, respectively. The completeness for the nf-HEDM measurements decreases near grain boundaries where the forward model projects diffraction to the edges of observed diffraction spots. Moreover, as the nf-HEDM reconstruction process is built on a forward model that currently does not include strain, distortions of the lattice result in lower completeness measured under load. The ff-HEDM completeness decreases with increasing deformation for several reasons, including a decrease in the above background intensity and "smearing" of the diffraction peaks due to increased defect content. It also becomes much more difficult to accurately segment individual diffraction signals once smearing becomes significant enough to cause a large degree of overlap. Efforts are underway to develop a truly integrated approach to the data analysis that treats the nf-HEDM and ff-HEDM measured data as a single X-ray experiment, as opposed to individual techniques with their different strengths and weaknesses.

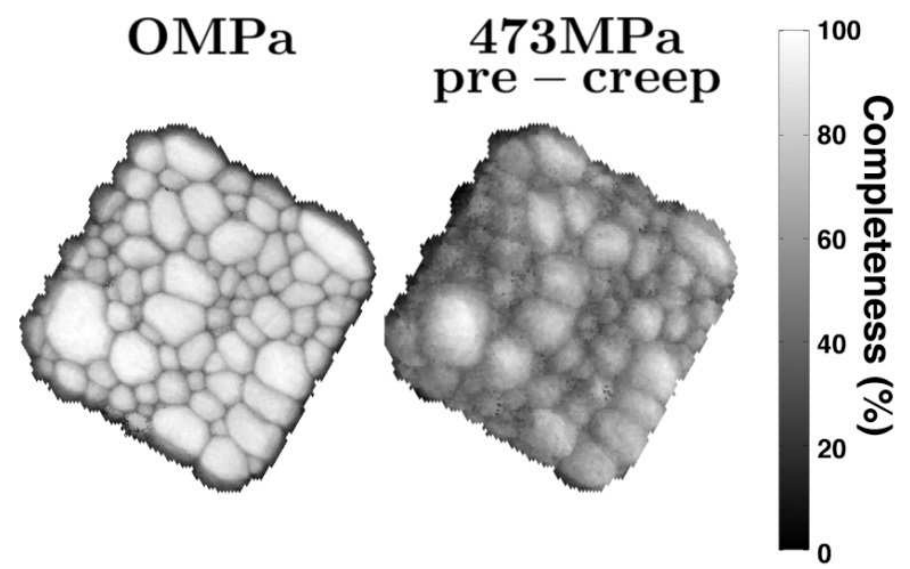

Figure 4. The completeness is shown as a percent for the nf-HEDM results. The results are shown using grayscale to highlight the variation in completeness surrounding grain boundaries.

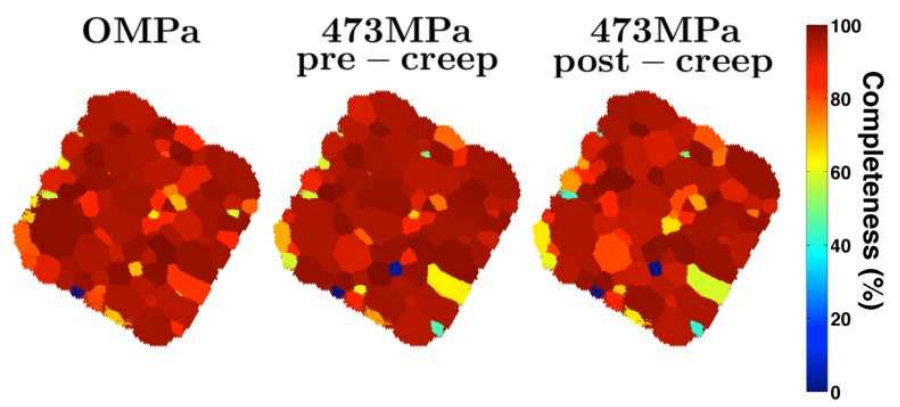

Figure 5. The completeness is shown as a percent for the ff-HEDM results. Given that the ffHEDM results are grain cross-section averaged, this color scale was selected for ease of viewing.

\subsection{Stress evolution}


Figure 6 shows grain cross-section averaged (GCSA) normal and shear stresses, obtained from ff-HEDM data, overlaid on the nf-HEDM grain map from Figure $2 b$. Here GCSA indicates the material response averaged over the grain cross section and the height of the beam ( 2 $\mu \mathrm{m})$. Using the typically quoted uncertainty for ff-HEDM lattice strain measurements of $\pm 1 \times 10^{-4}[19,20]$ and Young's modulus for titanium alloys (120 GPa), we approximate the uncertainty for each stress component as $\pm 12 \mathrm{MPa}$. Clearly, the GCSA stress states in different grains vary significantly. This is further illustrated in Figure 7, which shows scatter plots for each stress component versus grain ID and demonstrates an increase in scatter of the post creep deformation stress states. Comparisons between the lattice orientations in Figure $2 \mathrm{~b}$ and the stress maps of Figure 6 indicate that, while a portion of the heterogeneity is correlated with orientation, significant additional variation can be assigned to anisotropic local neighborhoods. This is particularly clear when one compares the before and after creep stresses, which are quite different in spite of the fixed loading condition. 
a
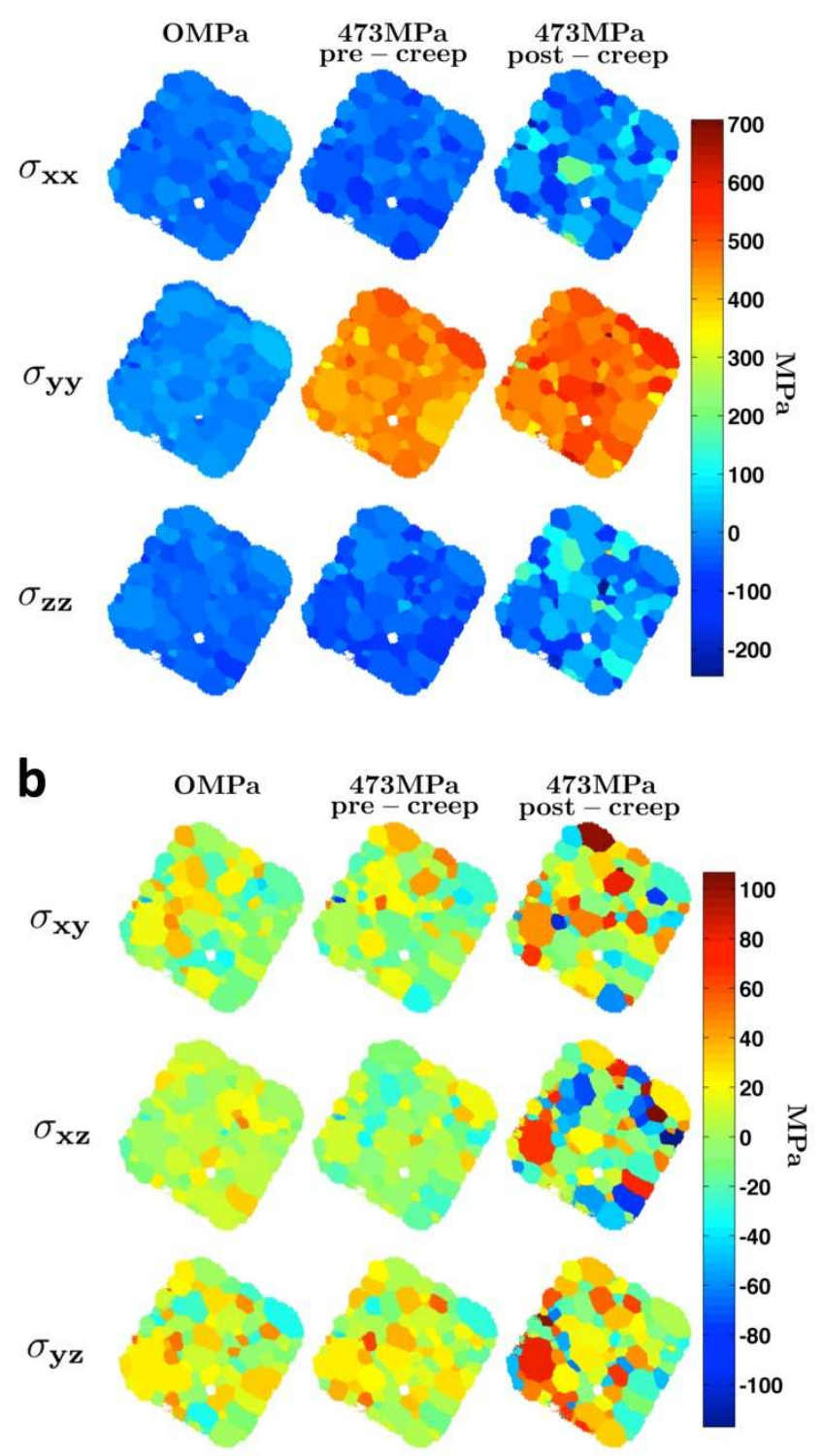

Figure 6. Distribution and evolution of stresses. Grain cross-section averaged (a), normal, and (b), shear, stresses measured with ff-HEDM for the specimen layer shown in Figure $2 \mathrm{~b}$. Loading is in the y-direction and results are shown at $0 \mathrm{MPa}$ before loading, at $473 \mathrm{MPa}$ prior to creep deformation, and at $473 \mathrm{MPa}$ after 24 hours of creep deformation. 

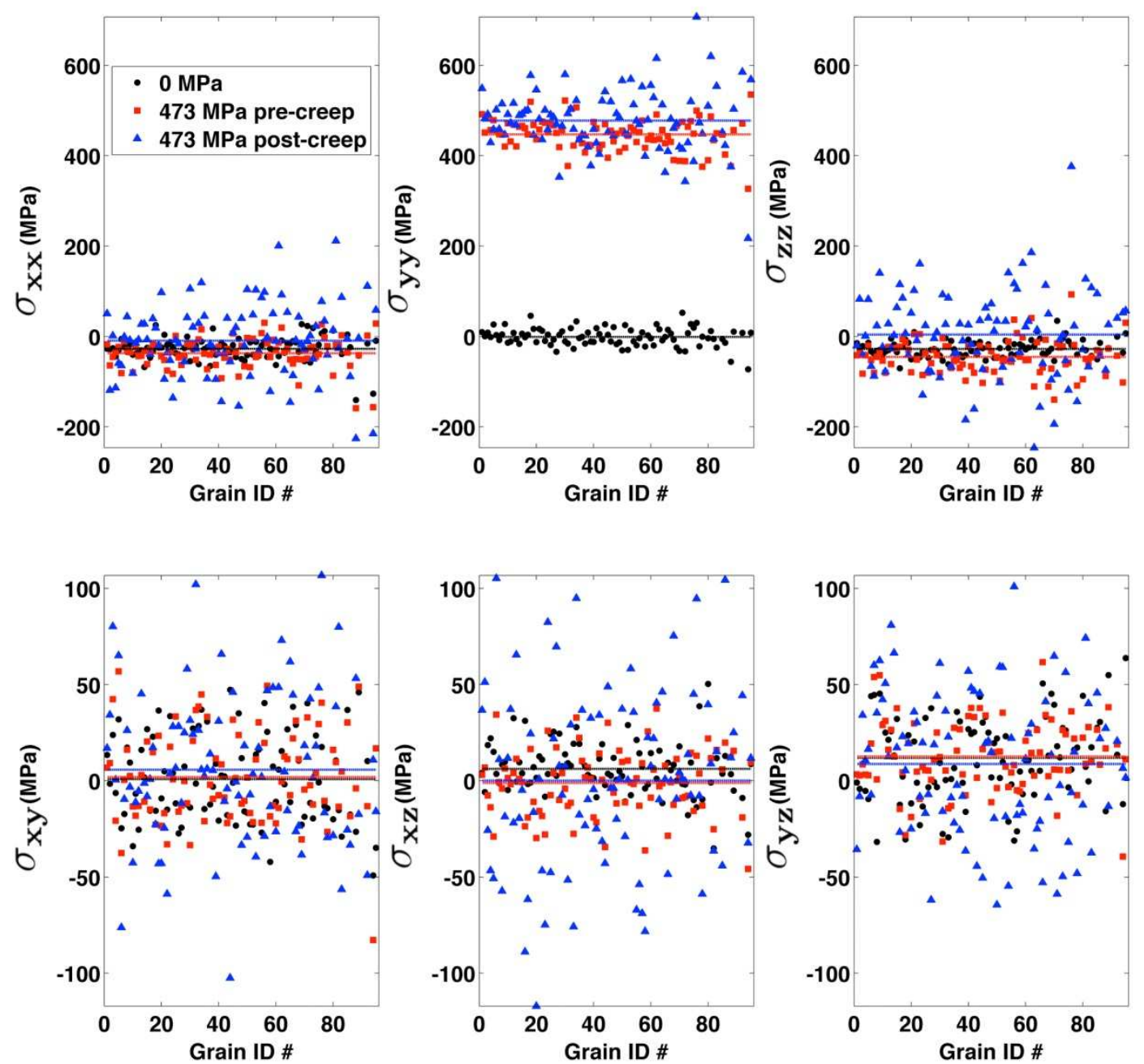

Figure 7. Stress scatter plots. The grain cross-section averaged stress data from Figure 6 is plotted against Grain ID, where the horizontal lines indicate the mean value for each deformation state. As one might expect, the mean values closely mirror the applied macroscopic stress state of uniaxial tension along the y-direction. Note the increased scatter with increasing deformation level.

Given that the body is in equilibrium, we know that the stresses within a cross-section must integrate to the applied stress state. Accepting that ff-HEDM provides GCSA quantities, we can approximate the integration by calculating a weighted average over the cross-section using the grain areas measured from the nf-HEDM analysis. The results for this weighted average are shown in Table 2. Though not conclusive, the close proximity of the stress state determined from the weighted average to the applied stress state provides increased confidence in the results. 


\begin{tabular}{lclllll}
\hline & $\sigma_{x x}$ & $\sigma_{y y}$ & $\sigma_{z z}$ & $\sigma_{x y}$ & $\sigma_{x z}$ & $\sigma_{y z}$ \\
\hline Zero Load & -25 & 2 & -27 & 2 & 7 & 9 \\
Pre Creep & -39 & 451 & -44 & 2 & -2 & 12 \\
Post Creep & -5 & 483 & 13 & 11 & -4 & 16
\end{tabular}

Table 2. The area weighted average of each stress component is provided in units of MPa. During the creep experiment the nonzero component of the applied macroscopic stress state $\left(\sigma_{y y}\right)$ was $473 \mathrm{MPa}$. The estimated uncertainty in each component is $\pm 12 \mathrm{MPa}$.

\subsection{Analysis}

The stress evolution in the grains is governed by the complex boundary value problem established by the anisotropic elastic and plastic properties of the individual grains within the mutual constraints of their local neighborhoods. Interestingly, we observe an increasingly heterogeneous stress field as creep proceeds. To quantitatively assess the stress evolution during creep, we focus on the von Mises effective stress $\left(\sigma_{e f f}\right)$ to describe deviatoric responses, the hydrostatic tension $\left(\sigma_{H}\right)$ for isotropic responses, and the stress co-axiality angle $(\phi)$. The stress co-axiality angle is defined as the angle between the stress vector for an individual grain, $\bar{\sigma}=\left[\sigma_{x x} \sigma_{y y} \sigma_{z z} \sigma_{x y} \sigma_{x z} \sigma_{y z}\right]$, and the applied macroscopic stress vector, in

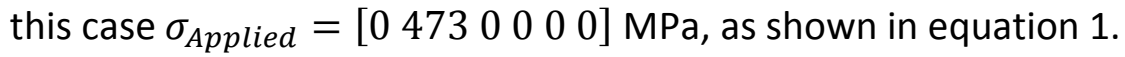

$$
\phi=\cos ^{-1} \frac{\bar{\sigma} \cdot \sigma_{\text {Applied }}}{|\bar{\sigma}|\left|\sigma_{\text {Applied }}\right|}
$$

This is a scalar quantity that conveys the rotation of the stress state during creep against which stresses can be readily plotted [28-30].

Figure 8a shows that while the mean GCSA effective stress across the displayed sample cross-section is nearly the same before and after creep deformation (498 MPa to $502 \mathrm{MPa}$ ), the standard deviation increases appreciably (32 MPa to $47 \mathrm{MPa}$ ). The largest relative positive and negative changes in the effective stress for individual grains are $145 \mathrm{MPa}$ and -109 $\mathrm{MPa}$, respectively. Conversely, the hydrostatic tension shown in Figure $8 \mathrm{~b}$ indicates a substantial increase in both the mean (123 MPa to $163 \mathrm{MPa}$ ) and standard deviation (23 MPa to $49 \mathrm{MPa}$ ). The largest change in the hydrostatic tension for individual grains was from $206 \mathrm{MPa}$ to 387 $\mathrm{MPa}$. Perhaps even more interesting is the evolution of the stress co-axiality angle shown in Figure 8c. Surprisingly, the stresses evolved away from the applied loading direction rather than toward it, indicating that during uniaxial creep deformation the intergranular stresses in the body trend toward greater triaxiality, as opposed to shifting to align with the applied load. Though not shown, we note that the modest creep deformation ( $0.6 \%$ plastic strain) does not lead to appreciable changes in average grain orientations or grain breakup associated with slip, indicating that the significant stress evolution cannot be explained through lattice rotations. We hypothesize that the creep evolution occurs through inhomogeneous and anisotropic motions of defects (dislocations) that transfer load between grains and account for the observed evolution of stress states. Since the applied load is constant but the time scale is extremely long by standards for thermally-activated or rate-dependent processes, these motions most likely correspond to depinning of dislocations and intermittent changes in stress state. Additional far- 
field HEDM data collected during the 24 hour creep period should shed considerable light on these rate-dependent dynamics.

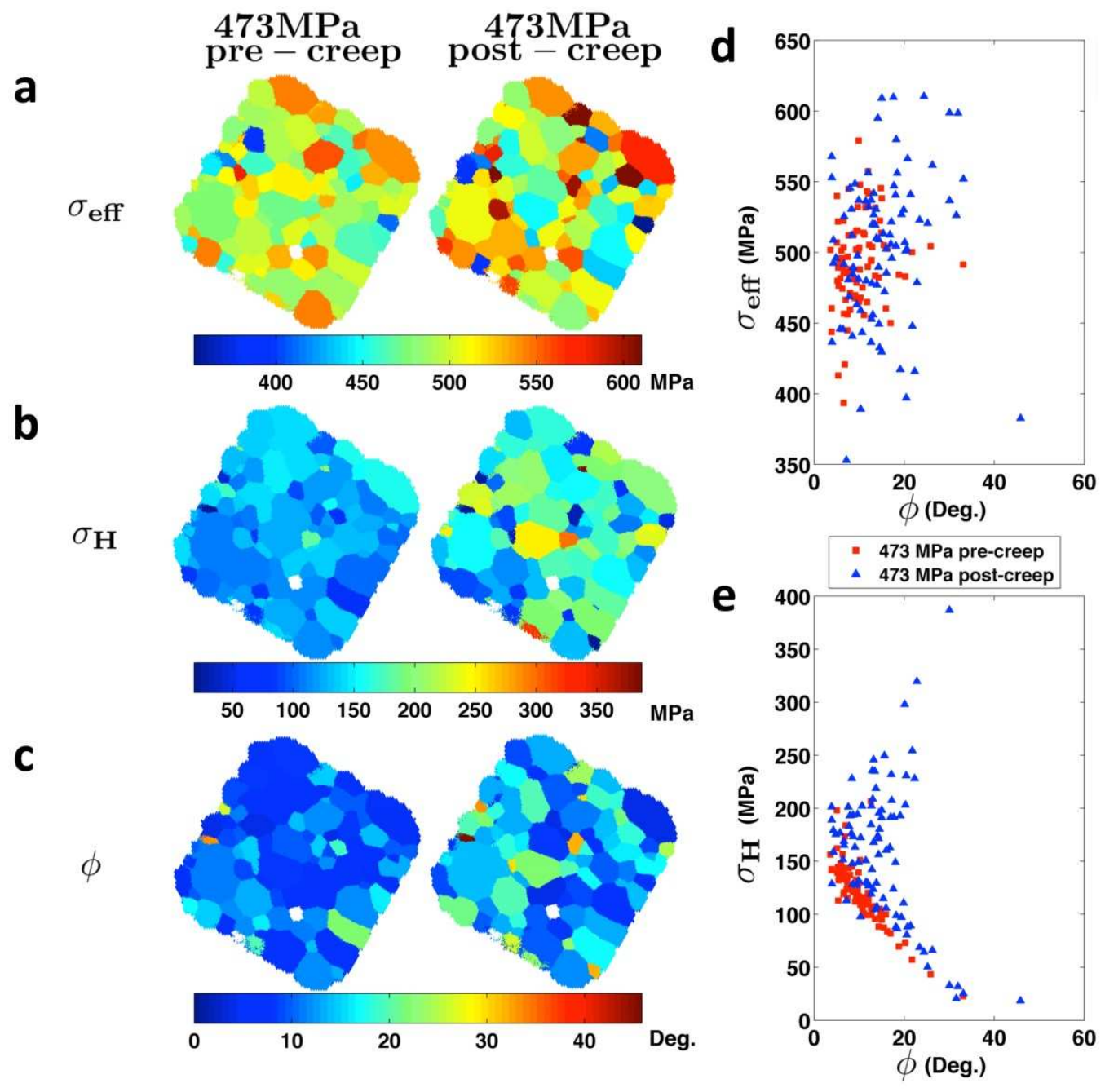

Figure 8. Analysis of stress evolution during creep deformation. Grain cross-section averaged (a), von Mises effective stress $\left(\sigma_{e f f}\right)$, (b), hydrostatic tension $\left(\sigma_{H}\right)$, and $(\mathbf{c})$, stress co-axiality angle $(\phi)$, shown before and after creep deformation at $473 \mathrm{MPa}$. (d), The effective stress and (e), hydrostatic tension, for each grain plotted against the stress co-axiality angle.

\subsection{Interpretation}

Though known within the mechanics community, it is worth stating the implications of these results on the commonly used Schmid factor analysis to indicate which slip systems will be active within a grain. This analysis, which was originally developed for interpreting deformation of single crystals [31], assumes the grain level stress state is uniaxial tension. The results presented herein, as well as in other ff-HEDM studies [32,33], show this assumption to be invalid for the polycrystalline specimen, particularly given the trend of increasing stress coaxiality angle during creep deformation shown in Figure 8c. 
The results in Figure 8e show a previously unknown phenomenon. Upon initial loading the hydrostatic tension decreases linearly with increasing stress co-axiality angle (negative slope of red points in Figure 8e). During the 24 hours at fixed applied load, this trend breaks down and grains with both the highest and lowest hydrostatic tension have a high stress coaxiality angle. Again, this phenomenon cannot be modeled on a single grain basis but rather must involve complex interactions.

The true value of the capability illustrated here lies in the opportunity to test hypotheses and explore complex phenomena that have historically restricted the development of mesoscale based design tools. For example, titanium alloys are prone to not only creep but also to the elusive phenomenon of dwell fatigue which compounds aspects of both static and cyclic loading and results in the propagation of a crack to failure. Dwell fatigue limits the lifetime of many aircraft components. Despite many research efforts, the underlying mechanisms remain unknown. Our data reveal the internal stresses in individual grains which result from complex grain interactions. Such data should offer significant insight into the mesoscale boundary conditions that drive crack initiation and short-crack growth. A large plastic strain is known to initiate void nucleation [34], and the voids are known to grow and coalesce when subjected to a high hydrostatic tensile stress [35,36], leading to a strong decrease in ductility with increasing hydrostatic tension [35,37-39]. Our analysis shows that initially high hydrostatic stress is correlated with low stress co-axiality angle. As the creep deformation proceeds the stress states become further decoupled from the external loading direction as seen by an increase in magnitude of the stress co-axiality angle (Figure 8e) and seemingly random spatial distribution (Figure 8c). This observation necessitates the development of validated mesoscale based models.

\subsection{Sources of error}

Though the results from this initial study are promising, these experiments contain many sources of error that influence the calculated stress values. Specifically, any error due to uncertainty in the lattice constants, elastic moduli, and the sample dimensions will result in a scaling of the stress values. Such errors would alter the values of the presented stresses, but not the nature of the trends.

Moreover, issues such as stage precision and detector distortions also come into play [40]. The propagation of errors in the fitted (gaussian assumption) diffraction spot positions through the least-squares fitting of the grain deformation gradient was discussed by Edmiston et al [41]. The values of the stress deviators have been shown to be relatively insensitive to small errors in the calibration of the instrument parameters. A detailed discussion of the calibration and deformation gradient fitting procedures may be found in [15]. It should also be noted again that the nf-HEDM and ff-HEDM scans were not measured concurrently in this study. Therefore it can be expected that the absolute registry of the diffracting volumes between the two scans could differ along the loading axis by $\sim 1 \mu \mathrm{m}$ (stage error, beam drift). Furthermore, small non-orthogonality between the rotation axis and the major axis of the beam profile could lead to very small portions of grains leaving the beam for some part of the $360^{\circ}$ scanning interval. This would lead to artificially low completeness values for otherwise valid grains. 


\subsection{Outlook}

Continuing developments within the HEDM community are rapidly advancing. Currently the data from each of the three techniques are analyzed separately and subsequently combined. Though the current datasets are key for model development, the quality will increase as we begin to adapt our treatment of the raw data to consider all three techniques in concert. Moving to a forward model of the interaction of the X-rays and the material that accounts for the signals measured at each detector will result in a tool that far exceeds the sum of the results from the conventional analysis for each technique. Moreover, such advances should cascade through other crystalline material systems. Our selected example highlights the strengths of the tool, however, for other materials such as ceramic matrix composites, the need for HEDM type datasets is equally pronounced given the complex interplay of evolving microstructure and void networks during loading [42,43].

We have shown here that three probes can be combined in one experimental setup: nfHEDM (grain geometries, orientations, orientation gradients, boundary networks), ff-HEDM (grain centers of mass, orientations, and strain states), $\mu-\mathrm{CT}$ (density variations due to inclusions, cracks, voids). The experimental facility at the APS 1-ID-E hutch and our in-situ mechanical loading system are also compatible with at least three additional configurations, each yielding complementary types of information. Instead of the single far-field detector used here, one can employ a four panel array of the same detector type (the "hydra" array) which yields increased strain resolution and/or higher q-coverage [44]. A "very far-field" configuration places an additional detector $\sim 4 \mathrm{~m}$ downstream of the sample; this allows high resolution reciprocal space mapping in the vicinity of isolated Bragg peaks which has been shown to yield information about nano-scale defect configurations such as dislocation cells. $[9,45,46]$. Finally, small angle scattering can be measured $\sim 6 \mathrm{~m}$ downstream of the sample to yield sensitivity to statistical distributions of nano-scale features such as second phases and early-stage voids $[44,47,48]$. It is possible to use any and all of these capabilities to probe an individual sample while under load. Further, a next generation RAMS device is being constructed that will facilitate cyclic and torsional loading at high temperatures. This extremely extensive and flexible set of capabilities promises to illuminate many mesoscale materials problems and should play an important role in development of improved understanding and modeling capabilities.

\section{Summary}

The nature of the application of structural materials demands that their performance be reliable. It's well known that the behavior of such materials is a product of the microstructure, and that failure initiation sites can often be linked to local microstructural features. Yet modern design and sustainment methodologies for structural materials remain reliant upon models with homogenized representations of the material structure and large-scale conventional mechanical testing efforts. This is extremely costly, both in the sense that conventional mechanical test databases are expensive to produce, and also that this homogenization approximation scheme inherently requires unnecessary conservatism as local microstructural (mesoscale) effects are ignored. Moving forward, the development and validation of a microstructure-sensitive modeling framework that can accurately predict materials behavior 
(including variability and uncertainty) would allow the maximization of component capability and life, while reducing cost/time to certify and improve safety.

The type of results presented here, with integrated data characterizing the material structure as well as internal stress distributions, should play a key role in developing a more sophisticated understanding of material behaviors and should serve as a basis for validation of modeling efforts. Development of new materials expressing improved performance characteristics, as well as building efficiencies into the design process, rests upon establishing validated computational models that link materials processing to microstructure and microstructure to properties and performance [49]. Trusted modeling tools for the prediction of responses at the grain level and above have been severely limited by the historical lack of in situ characterization at the mesoscale.

We have demonstrated a new experimental capability that provides a micron scale (mesoscale) spatiotemporally-resolved probe for tracking evolving microstructure correlated with the stresses driving deformation. These first-of-a-kind results already show an unexpected evolution of grain level stresses in a titanium alloy undergoing creep deformation. We anticipate many additional discoveries since this new form of microscopy is in its infancy. Within this powerful new methodology, the explicit microstructure becomes the test bed for exploring the validity of historically necessary or wholly new assumptions, and for driving further model development. Moreover, these datasets open the possibility of new fundamental design frameworks built upon quantitatively defined hierarchies of theories coupling nano-scale to meso-scale and macroscopic responses. Such models are critical for developing the next generation of engineering design protocols, for example the design of components with graded microstructures, where the microstructure at a specific point in a component is tailored to provide optimized properties for that location.

\section{References}

[1] McDowell DL, et al. Integrated design of multiscale, multifunctional materials and products. Burlington: Butterworth-Heinemann; 2010.

[2] US Department of Energy Basic Energy Science Report. From quanta to the continuum: opportunities for mesoscale science. 2012.

[3] Miller MP, et al. High-energy needs and capabilities to study multiscale phenomena in crystalline materials. Synchrotron Radiation News 2012;25:18-26.

[4] Committee on Integrated Computational Materials Engineering, National Research Council. Integrated computational materials engineering: a transformational discipline for improved competitiveness and national security. Washington D.C.: National Academies Press; 2008.

[5] Bernal JD. On the interpretation of X-ray, single crystal, rotation photographs. Proc Roy Soc Lond A 1926;113:117-160.

[6] Kabsch W. Evaluation of single-crystal X-ray diffraction data from a position-sensitive detector. J Appl Cryst 1988;21:916-924.

[7] Lauridsen EM, Schmidt S, Suter RM, Poulsen HF. Tracking: a method for structural characterization of grains in powders or polycrystals. J Appl Cryst 2001;34:744-750.

[8] Poulsen HF. Three-dimensional X-Ray diffraction microscopy: mapping polycrystals and their dynamics. Berlin: Springer; 2004. 
[9] Lienert $U$, et al. High-energy diffraction microscopy at the Advanced Photon Source. JOM 2011;63:70-77.

[10] Poulsen HF. An introduction to three-dimensional X-ray diffraction microscopy. J Appl Cryst 2012;45:1084-1097.

[11] Larson BC, Yang W, Ice GE, Budai JD, Tischler JZ. Three-dimensional X-ray structural microscopy with submicrometre resolution. Nature 2002;415:887-890.

[12] Poulsen HF, et al. Three-dimensional maps of grain boundaries and the stress state of individual grains in polycrystals and powders. J Appl Cryst 2001;34:751-756.

[13] Margulies L, Lorentzen T, Poulsen HF, Leffers T. Strain tensor development in a single grain in the bulk of a polycrystal under loading. Acta Mater 2002;50:1771-1779.

[14] Oddershede J, et al. Determining grain resolved stresses in polycrystalline materials using three-dimensional X-ray diffraction. J Appl Cryst 2010;43:539-549.

[15] Bernier JV, Barton NR, Lienert U, Miller MP. Far-field high-energy diffraction microscopy: a tool for intergranular orientation and strain analysis. J Strain Analysis Eng Des 2011;46:527-547.

[16] Suter RM, Hennessy D, Xiao C, Lienert U. Forward modeling method for microstructure reconstruction using $X$-ray diffraction microscopy: single crystal verification. Rev Sci Instrum 2006;77:123905.

[17] Li SF, Suter RM. Adaptive reconstruction method for three-dimensional orientation imaging. J Appl Cryst 2013;46:512-524.

[18] Withers PJ, Preuss M. Fatigue and damage in structural materials studied by X-ray tomography. Annu Rev Mater Res 2012;42:81-103.

[19] Schuren JC, Miller MP. Quantifying the uncertainty of synchrotron-based lattice strain measurements. J Strain Analysis Eng Des 2011;46:663-681.

[20] Schuren JC, Wong SL, Dawson PR, Miller MP. Integrating experiments and simulations to estimate uncertainty in lattice strain measurements. J Strain Analysis Eng Des 2014;49:33-50.

[21] Blank BE, Schuren J, Shade P, Turner T. Rotational and axial motion system and methods of use. US Patent Application 14/461,582; filed 08/18/2014.

[22] Fisher ES, Renken CJ. Single-crystal elastic moduli and the hcp-bcc transformation in Ti, Zr, and Hf. Phys Rev 1964;135:482-494.

[23] Imam MA, Gilmore CM. Room temperature creep of Ti-6Al-4V. Metall Trans A 1979;10:419-425.

[24] Bache MR. A review of dwell sensitive fatigue in titanium alloys: the role of microstructure, texture and operating conditions. Int J Fatigue 2003;25:1079-1087.

[25] Brandes MC, Mills MJ, Williams JC. The influence of slip character on the creep and fatigue fracture of an $\alpha$ Ti-Al alloy. Metall Mater Trans A 2010;41:3463-3472.

[26] Pilchak AL, Williams JC. Observations of facet formation in near- $\alpha$ titanium and comments on the role of hydrogen. Metall Mater Trans A 2011;42:1000-1027.

[27] Frank FC. Orientation mapping. Metall Trans A 1988;19:403-408.

[28] Yamaji A, Sato K. Distances for the solution of stress tensor inversion in relation to misfit angles that accompany the solutions. Geophysics J International 2006;167:933-942. 
[29] Ritz H, Dawson P, Marin T. Analyzing the orientation dependence of stresses in polycrystals using vertices of the single crystal yield surface and crystallographic fibers of orientation space. J Mech Phys Solids 2009;58:54-72.

[30] Turner TJ, Shade PA, Schuren JC, Groeber MA. The influence of microstructure on surface strain distributions in a nickel micro-tension specimen. Modelling Simul Mater Sci Eng 2013;21:015002.

[31] Schmid E, Boas W. Plasticity of crystals. Berlin: Springer; 1935.

[32] Bernier JV, Miller MP. A direct method for the determination of the mean orientationdependent elastic strains and stresses in polycrystalline materials from strain pole figures. J Appl Cryst 2006;39:358-368.

[33] Bernier JV, Park JS, Pilchak AL, Glavicic MG, Miller MP. Measuring stress distributions in Ti-6Al-4V using synchrotron X-ray diffraction. Metall Mater Trans A 2008;39:3120-3133.

[34] Goods SH, Brown LM. Overview no. 1: the nucleation of cavities by plastic deformation. Acta Metall 1979;27:1-15.

[35] Rice JR, Tracey DM. On the ductile enlargement of voids in triaxial stress fields. J Mech Phys Solids 1969;17:201-217.

[36] Barton NR, Dawson PR. Lattice misorientations in titanium alloys: modeling the origins of defects. IJFP-5 Materials Processing Defects 2002;191-203.

[37] McClintock FA. A criterion for ductile fracture by the growth of holes. J Appl Mech 1968;35:363-371.

[38] Tvergaard V, Needleman A. Analysis of the cup-cone fracture in a round tensile bar. Acta Metall 1984;32:157-169.

[39] Mirza MS, Barton DC, Church P. The effect of stress triaxiality and strain-rate on the fracture characteristics of ductile metals. J Mater Sci 1996;31:453-461.

[40] Borbely A, Renbersade L, Kenesei P, Wright J. On the calibration of high-energy X-ray diffraction setups. I. Assessing tilt and spatial distortion of the area detector. J Appl Cryst 2014;47:1042-1053.

[41] Edmiston JK, Barton NR, Bernier JV, Johnson GC, Steigmann DJ. Precision of lattice strain and orientation measurements using high-energy monochromatic X-ray diffraction. J Appl Cryst 2011;44:299-312.

[42] Morales-Rodriguez A, Reynaud P, Fantozzi G, Adrien J, Maire E. Porosity analysis of longfiber reinforced ceramic matrix composites using X-ray tomography. Scripta Mater 2009;60:388-390.

[43] Bale HA, et al. Real-time quantitative imaging of failure events in materials under load at temperatures above 1,600 C. Nature Mater 2013;12:40-46.

[44] Wang L, Li M, Almer J. In situ characterization of Grade 92 steel during tensile deformation using concurrent high energy X-ray diffraction and small angle X-ray scattering. J Nucl Mater 2013;440:81-90.

[45] Jakobsen $B$, et al. Formation and subdivision of deformation structures during plastic deformation. Science 2006;312:889-892.

[46] Wejdemann C, Poulsen HF, Lienert U, Pantleon W. In situ observation of the dislocation structure evolution during a strain path change in copper. JOM 2013;65:35-43. 
[47] Wang XL, et al. In situ synchrotron study of phase transformation behaviors in bulk metallic glass by simultaneous diffraction and small angle scattering. Phys Rev Lett 2003;91:265501.

[48] Almer JD, Stock Sr. Micromechanical response of mineral and collagen phases in bone. J Struct Biol 2007;157:365-370.

[49] Executive Office of the President, National Science and Technology Council. Materials genome initiative for global competitiveness. 2011.

\section{Acknowledgements}

The authors would like to thank Dr. Adam Pilchak (Air Force Research Laboratory) for providing the Ti-7Al material examined in this study, Dr. Chris Woodward (Air Force Research Laboratory) for help securing the computational resources required for the data reduction, Dr. Andrew Rosenberger (Air Force Research Laboratory) for providing reference mechanical test data, Professor Armand Beaudoin (University of Illinois) and Professor Matt Miller (Cornell University) for useful discussions, and Ali Mashayekhi (Advanced Photon Source), Erika Benda (Advanced Photon Source), and Kurt Goetze (Advanced Photon Source) for help with the experimental setup. The authors acknowledge support from the Materials \& Manufacturing Directorate of the U.S. Air Force Research Laboratory. Use of the Advanced Photon Source, an Office of Science User Facility operated for the U.S. Department of Energy (DOE) Office of Science by Argonne National Laboratory, was supported by the U.S. DOE under Contract No. DEAC02$06 \mathrm{CH} 11357$. 